\title{
Prognostic and Clinicopathological Value of Programmed Death Ligand-1 in Breast Cancer: A Meta-Analysis
}

\author{
Yawen Guo ${ }^{\ddagger}$, Pan $\mathrm{Yu}^{\ddagger \oplus}$, Zeming Liu, Yusufu Maimaiti, Shan Wang, Xingjie Yin, \\ Chunping Liu ${ }^{\ddagger *}$, Tao Huang ${ }^{\ddagger *}$
}

Department of Breast and Thyroid Surgery, Union Hospital, Tongji Medical College, Huazhong University of Science And Technology, Wuhan, China

ه These authors contributed equally to this work. $\ddagger Y G$ and $P Y$ are first authors on this work. CL and TH also contributed equally to this work as corresponding authors.

* huangtaowh@163.com (TH); Icp191@ 163.com (CL)

\section{Gopenaccess}

Citation: Guo Y, Yu P, Liu Z, Maimaiti Y, Wang S, Yin $X$, et al. (2016) Prognostic and Clinicopathological Value of Programmed Death Ligand-1 in Breast Cancer: A Meta-Analysis. PLoS ONE 11(5): e0156323. doi:10.1371/journal.pone.0156323

Editor: Elda Tagliabue, Fondazione IRCCS Istituto Nazionale dei Tumori, ITALY

Received: February 5, 2016

Accepted: May 12, 2016

Published: May 26, 2016

Copyright: @ 2016 Guo et al. This is an open access article distributed under the terms of the Creative Commons Attribution License, which permits unrestricted use, distribution, and reproduction in any medium, provided the original author and source are credited.

Data Availability Statement: All relevant data are within the paper and its Supporting Information files.

Funding: This work was supported by grants from the the National Natural Science Foundation of China: NSFC 81550009 (http://www.nsfc.gov.cn/). The funders had no role in study design, data collection and analysis, decision to publish, or preparation of the manuscript.

Competing Interests: The authors have declared that no competing interests exist.

\section{Abstract}

Recently, the interest in programmed death ligand-1 (PD-L1) as a prognostic marker in several types of malignant tumors has increased. In the present meta-analysis, we aimed to explore the prognostic and clinicopathological value of PD-L1 in breast cancer. We searched Medline/PubMed, Web of Science, EMBASE, the Cochrane Library databases, and grey literature from inception until January 20, 2016. Studies concerning breast cancer that focused on PD-L1 expression and studies reporting survival data were included; two authors independently performed the data extraction. The pooled risk ratio (RR) and 95\% confidence interval $(\mathrm{Cl})$ were assessed to determine the association between the clinicopathological parameters of patients and PD-L1 expression. Five studies involving 2061 patients were included in this meta-analysis. The results indicated that positive/higher PDL1 expression was a negative predictor for breast cancer, with an RR of $1.64(95 \% \mathrm{Cl}$, 1.14-2.34) for the total mortality risk and an RR of $2.53(95 \% \mathrm{Cl}, 1.78-3.59)$ for the mortality risk 10 years after surgery. Moreover, positive/higher PD-L1 expression was significantly associated with positive lymph node metastasis (RR, 1.33; 95\% Cl, 1.04-1.70), poor nuclear grade (RR, 1.24; $95 \% \mathrm{Cl}, 1.07-1.43$ ), and negative estrogen receptor status (RR, $2.45 ; 95 \% \mathrm{Cl}, 1.31-4.60)$ in breast cancer patients. Our findings suggest that PD-L1 can serve as a significant biomarker for poor prognosis and the adverse clinicopathologic features of breast cancer and could facilitate the better management of individual patients.

\section{Introduction}

Breast cancer is currently the most frequently diagnosed cancer and is the leading cause of cancer-related death in women; in fact, in 2012, a total of 1.7 million new cases breast cancer and 521,000 cases of breast cancer-related mortality were reported worldwide [1]. At present, the prognosis, classification, and treatment of breast cancer is dependent on tumor histological grading, lymph node stage, and tumor stage (TNM), as well as 3 major protein markers: 
estrogen receptor (ER), progesterone receptor (PR), and human epidermal growth factor (EGF) receptor 2 (HER2) [2, 3]. However, breast cancer is generally not considered an immunogenic malignancy. Even though breast cancer cells use immune pathways to evade antitumor immune responses and progressively grow and metastasize, no treatment that enhances the antitumor immune response is currently used [4]. However, some investigators have begun to focus on novel immunotherapeutic strategies for treating breast cancer.

The expression of the programmed cell death 1 (PD-1), a member of the B7 family of immune-regulatory cell-surface proteins, and its cognate ligand PD-L1, within the tumor microenvironment is a major resistance mechanism for escaping immune surveillance $[5,6]$. PD-L1 is expressed in tumor-infiltrating lymphocytes and tumor cells of cancer including breast, lung, prostate, gastrointestinal, and malignant melanomas [7-11]. Furthermore, PD-L1 expression is associated with poor prognosis in breast, pancreatic, and renal cell cancers [1218]. To better understand the potential relationship between PD-L1 and prognosis in breast cancer, it should be clarified whether PD-L1 is a possible target for the treatment of breast cancer.

Although some studies have been conducted to analyze the relationship between PD-L1 and breast cancer, its prognostic role in breast cancer remains controversial. To our knowledge, no meta-analyses have been performed on this topic thus far. In this study, we aimed to perform an up-to-date meta-analysis to determine the prognostic value of PD-L1 in breast cancer.

\section{Methods}

This study was conducted and reported according to the Preferred Reporting Items for Systematic Reviews and Meta-Analyses (PRISMA) statement checklist (S1 File).

\section{Data search strategy}

We searched the Medline/PubMed, Web of Science, EMBASE, the Cochrane Library databases, and grey literature from inception January 20, 2016. The search strategy used both MeSH terms and free-text words to increase sensitivity. The key terms employed for literature retrieval included "PD-L1," "programmed death ligand-1," "CD274," "B7-H1," or "B7 homo$\log 1$ "; "breast cancer," "breast carcinoma," or "breast tumor"; and "survival," "outcome," or "prognosis. We also contacted the corresponding authors to obtain any additional information, if necessary.

\section{Inclusion and exclusion criteria}

Articles were selected if they met the following criteria: (i) they were focused on breast cancer; (ii) all selected cancer patients were confirmed as having breast cancer via pathological examination; and (iii) the correlation between PD-L1, clinicopathological features, and prognosis was discussed. Studies were excluded if they met any of the following criteria: (i) duplicate publication; (ii) non-human experiments were performed, non-English papers; (iii) conference abstract; (iv) review articles, case reports, or letters; or (v) insufficient data regarding 95\% confidence interval (95\% CI) and risk ratios (RR) provided, or (vi) the Kaplan-Meier curve could not be extracted. In cases where more than one article was published from the same center, the study with the information most relevant to the present study was included.

\section{Data extraction}

Two independent reviewers (Yawen Guo and Pan Yu) extracted all the data, and the following information was recorded: author, year of publication, patient number, country, specimen, 
detection method, cut-off values for the positive rates of PD-L1 overexpression, duration of follow-up after surgery, study end points, and data presented in the tables and figures. For articles that only provided survival data in a Kaplan Meier curve, the survival rates were calculated using Engauge Digitizer software, version 3.0 (http://digitizer.sourceforge.net) to reconstruct the RR estimate and its variance, assuming that patients were censored at a constant rate during follow-up. The quality of the selected articles was assessed according to the Newcastle Ottawa Scale [19].

\section{Statistical analysis}

Statistical analysis was performed according to the guidelines proposed by the MetaAnalysis of Observational Studies in Epidemiology (MOOSE) group [20]. Data from each study were analyzed using Review Manager software, version 5.3 (Copenhagen: The Nordic Cochrane Centre, The Cochrane Collaboration, 2014) and Stata SE12.0 (Stata Corporation, TX, USA). Funnel plots were used to assess publication bias, and $p$ values of $<0.05$ were considered statistically significant. An RR of $>1$ indicated worse survival for patients with high PD-L1 expression, whereas an RR of $<1$ implied a survival benefit. The RRs and their 95\% CIs were used to assess the correlations between PD-L1 expression and the clinicopathological features of breast cancer, including tumor size; TNM stage; nuclear grade; lymph node metastasis; and the expression of ER, PR, Her-2, and Ki67. The pooled RR of each study was calculated using a fixedeffects model if there was no significant heterogeneity occurred among the studies, whereas a random-effects model was adopted if heterogeneity was observed. The heterogeneity among the data was evaluated by using the chi-square test and the $\mathrm{I}^{2}$ statistic. An $\mathrm{I}^{2}$ value of $>50 \%$ of the $\mathrm{I}^{2}$ statistic was considered to indicate significant heterogeneity [21]. All the p values were two-tailed.

\section{Results}

\section{Search results}

We identified 376 articles using our search strategy (S2 File). After screening the titles and abstracts, we excluded 291 articles because they were not original articles (e.g., review, letter, case report), not breast cancer-related studies, not English language papers, not human studies, or were conference abstracts. After reviewing the complete text of 85 articles, 80 articles were excluded because some of them were review articles, case reports, or letters; while some articles did not have sufficient data regarding the $95 \%$ confidence interval (CI) and risk ratios (RRs), or the Kaplan Meier data could not be extracted, leaving only 5 studies involving 2061 patients that were included in the meta-analysis. The details of the screening procedure are illustrated in Fig 1. All the enrolled articles comprehensively assessed the expression of PD-L1, clinicopathological features of breast cancer, and survival rate.

\section{Study selection and characteristics}

The details of the 5 eligible studies are listed in Table 1 [13-17]. The publication years of the eligible studies ranged from 2014 to 2016, and the number of patients in each study ranged from 36 to 870 . The PD-L1 expression levels were measured in tumor tissues in all 5 studies, including 4 studies wherein tissue immunochemical staining (IHC) was used to detect PD-L1 expression and 1 study wherein DNA contents (determined by flow cytometry) were used to survey the genomes of each tumor. As indicated in Table 1, each article had a specific cut-off value, which consequently influenced the positive rates of PD-L1 overexpression in each study. Moreover, the mean duration of follow-up after surgery ranged from 4.7 to 9.8 years. The 


\section{PRISMA 2009 Flow Diagram}
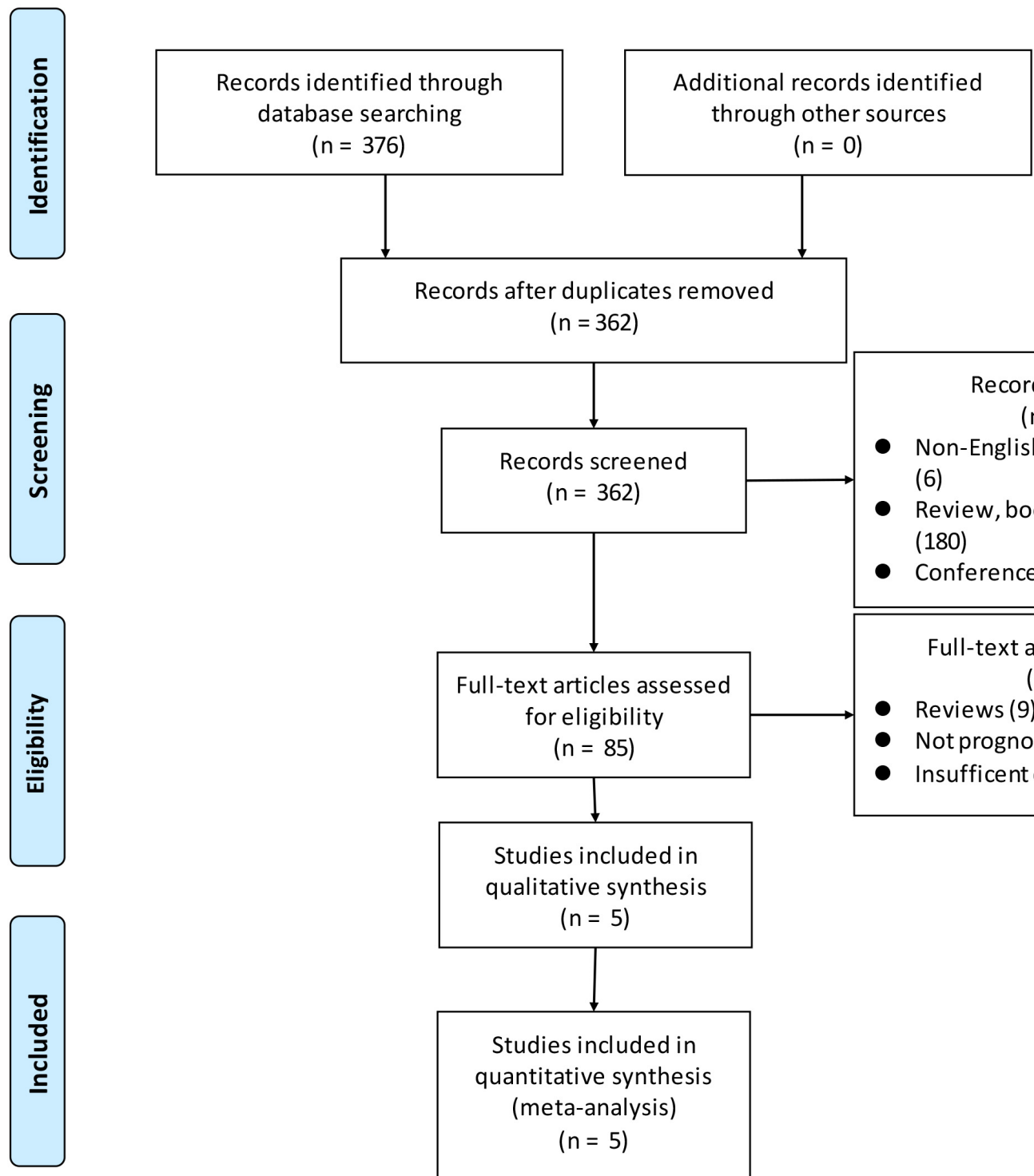
for eligibility

Records excluded $(n=277)$

- Non-English, non-human studies (6)

- Review, book, letter, case report (180)

- Conference abstract (91)

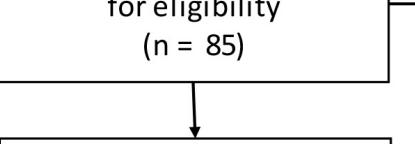

Full-text articles excluded $(n=80)$

- Reviews (9)

- Not prognosis study (61)

- Insufficent data(10)

Studies included in qualitative synthesis $(n=5)$

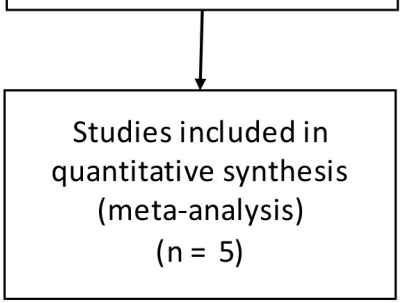

From: Moher D, Liberati A, Tetzlaff J, Altman DG, The PRISMA Group (2009). Preferred Reporting /tems for Systematic Review s and MetaAnalyses: The PRISMA Statement. PLoS Med 6(7): e1000097. doi:10.1371/journal.pmed1000097

For more information, visit www.prisma-statement.org.

Fig 1. Flowchart of the study selection process.

doi:10.1371/journal.pone.0156323.g001 
Table 1. Main characteristics of the studies included in this meta-analysis.

\begin{tabular}{|c|c|c|c|c|c|c|c|c|}
\hline $\begin{array}{l}\text { First author of } \\
\text { study }\end{array}$ & Year & $\begin{array}{l}\text { Number of } \\
\text { patients }\end{array}$ & Country & Specimen & Detection method & $\begin{array}{c}\text { Cut-off (positive/High } \\
\text { expression) }\end{array}$ & $\begin{array}{l}\text { Follow up } \\
\text { (years) }\end{array}$ & $\begin{array}{l}\text { End } \\
\text { point }\end{array}$ \\
\hline Muenst & 2014 & 650 & Switzerland & Tissue & $\mathrm{IHC}$ & H-Score $\geq 100(23.4 \%)$ & $\begin{array}{c}5.4(0.08- \\
14.5)\end{array}$ & OS \\
\hline Barrett & 2015 & 36 & America & Tissue & $\begin{array}{l}\text { DNA content Flow } \\
\text { cytometry }\end{array}$ & $\begin{array}{l}\text { High level }(\log 2 \text { ratio } \geq 1) \\
\text { amplicon }(22.2 \%)\end{array}$ & $\begin{array}{c}4.7(0.9- \\
12.0)\end{array}$ & $\begin{array}{c}\text { DFS/ } \\
\text { OS }\end{array}$ \\
\hline Park & 2015 & 316 & Korea & Tissue & $\mathrm{IHC}$ & H-Score $\geq 3+(51.6 \%)$ & $\begin{array}{l}9.8(0.4- \\
12.8)\end{array}$ & $\begin{array}{l}\text { DFS/ } \\
\text { OS }\end{array}$ \\
\hline Qin & 2015 & 870 & China & Tissue & $\mathrm{IHC}$ & $\begin{array}{c}\geq 5 \% \text { tumor cell staining } \\
(21.7 \%)\end{array}$ & $\begin{array}{l}8.2(1.4- \\
22.1)\end{array}$ & $\begin{array}{c}\text { DFS/ } \\
\text { OS }\end{array}$ \\
\hline Baptista & 2016 & 189 & Brazil & Tissue & $\mathrm{IHC}$ & Median (56.6\%) & $7.18^{1}$ & $\begin{array}{c}\text { DFS/ } \\
\text { OS }\end{array}$ \\
\hline
\end{tabular}

${ }^{1}$ median

DFS, disease-free survival; $\mathrm{H}$-score, Histo-score; OS, overall survival

doi:10.1371/journal.pone.0156323.t001

Disease-free survival (DFS) and overall survival (OS) were used as end points in 4 studies, whereas OS alone was used in 1 study as the end point (Table 1). Furthermore, the clinicopathological features including tumor size; TNM stage; nuclear grade; lymph node metastasis; and expression of ER, PR, Her-2, and Ki67 were reported in all 5 studies.

\section{Main results}

As noted in Fig 2, positive PD-L1 expression significantly associated with enhanced total mortality risk (MR) among breast cancer patients in the random-effects model; the pooled RR was 1.64 (95\% CI, 1.14-2.34), despite the presence of heterogeneity among the studies $\left(\mathrm{I}^{2}=85 \%\right.$,

A

PD-L1+ PD-L1- Risk Ratio

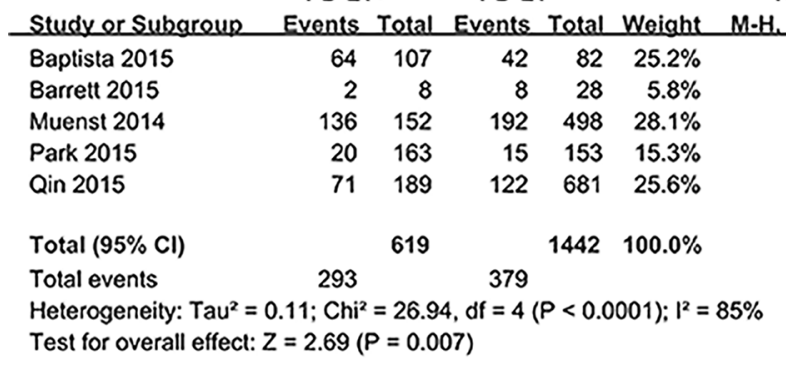

B Study or Subgreup Events Total Events Tota Baptista 2015 Muenst 2014 Park 2015 Qin 2015

Total $(95 \% \mathrm{Cl})$

Tolal events

Heterogeneity: $\mathrm{Tau}^{2}=0.09 ; \mathrm{Chi}^{2}=15.49, \mathrm{df}=3(P=0.001) ; \mathrm{I}^{2}=81 \%$

Test for overall effect: $Z=5.21(P<0.00001)$
Risk Ratio Risk Ratio

$1.17(0.90,1.52]$

$0.88[0.23,3.33]$

$2.32[2.05,2.63]$

$1.25[0.67,2.35]$

$2.10[1.64,2.68]$

$1.64[1.14,2.34]$
M.H. Randem. $25 \% \mathrm{Cl}$

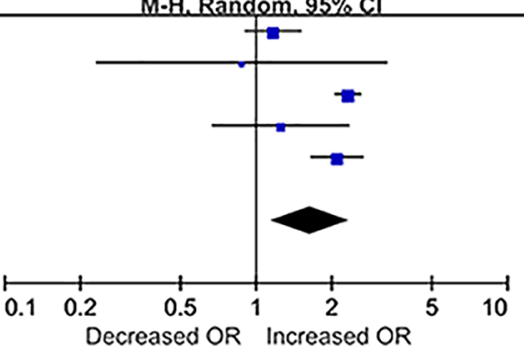

Decreased OR Increased OR

Risk Ratio M.H. Randem. $25 \%$

M-H. Randem. $95 \% \mathrm{Cl}$ $3.27[2.02,5.30]$

$2.32[2.05,2.63]$

$1.23[0.67,2.27]$

$3.52[2.72,4.55]$

$2.53[1.78,3.59]$

Fig 2. Forest plots of studies evaluating risk ratios (RRs) of PD-L1 for breast cancer specific survival. (A) Total mortality risk (MR) among breast cancer patients. (B) The MR 10 years after surgery (MR Meyears $)$ in breast cancer patients.

doi:10.1371/journal.pone.0156323.g002 
$\mathrm{p}<0.0001$; Fig 2A). Due to differences in the follow-up duration of each study, we assessed the mortality risk 10 years after surgery $\left(\mathrm{MR}_{10 \text { years }}\right)$ using a random-effects model and data from 4 articles with sufficient data. The RR was 2.53 (95\% CI, 1.78-3.59), despite the presence of significant heterogeneity among the studies $\left(\mathrm{I}^{2}=81 \%, \mathrm{p}=0.001\right.$; Fig $\left.2 \mathrm{~B}\right)$. Due to the presence of significant heterogeneity in the mortality risk across the studies, we further examined the potential sources of heterogeneity through metaregression, and found that the year of publication, detection method, and analysis method did not contribute to the heterogeneity.

Moreover, we assessed the relationship between positive PD-L1 expression and clinicopathological features (Fig 3). Lymph node metastasis of breast cancer was reported in all 5 studies. Due to the presence of significant heterogeneity $\left(\mathrm{I}^{2}=80 \%, \mathrm{p}=0.0006\right)$, the randomeffect model was adopted, which indicated a pooled RR (PD-L1-positive versus PD-L1-negative) of 1.33 (95\% CI, 1.04-1.70; Fig 3A). In addition, 4 studies reported on the nuclear grade of breast cancer. Due to the presence of significant heterogeneity $\left(\mathrm{I}^{2}=56 \%, \mathrm{p}=0.08\right)$, a randomeffects model was adopted, which indicated a pooled RR ( 3 versus $\leq 2)$ of 1.24 ( $95 \%$ CI, 1.07 1.43; Fig 3B). Furthermore, 3 studies reported on the ER status of breast cancer. Here as well, due to the presence of significant heterogeneity $\left(\mathrm{I}^{2}=94 \%, \mathrm{p}<0.00001\right)$, the random-effect model was adopted, which indicated a pooled RR (PD-L1-negative versus PD-L1-positive) of

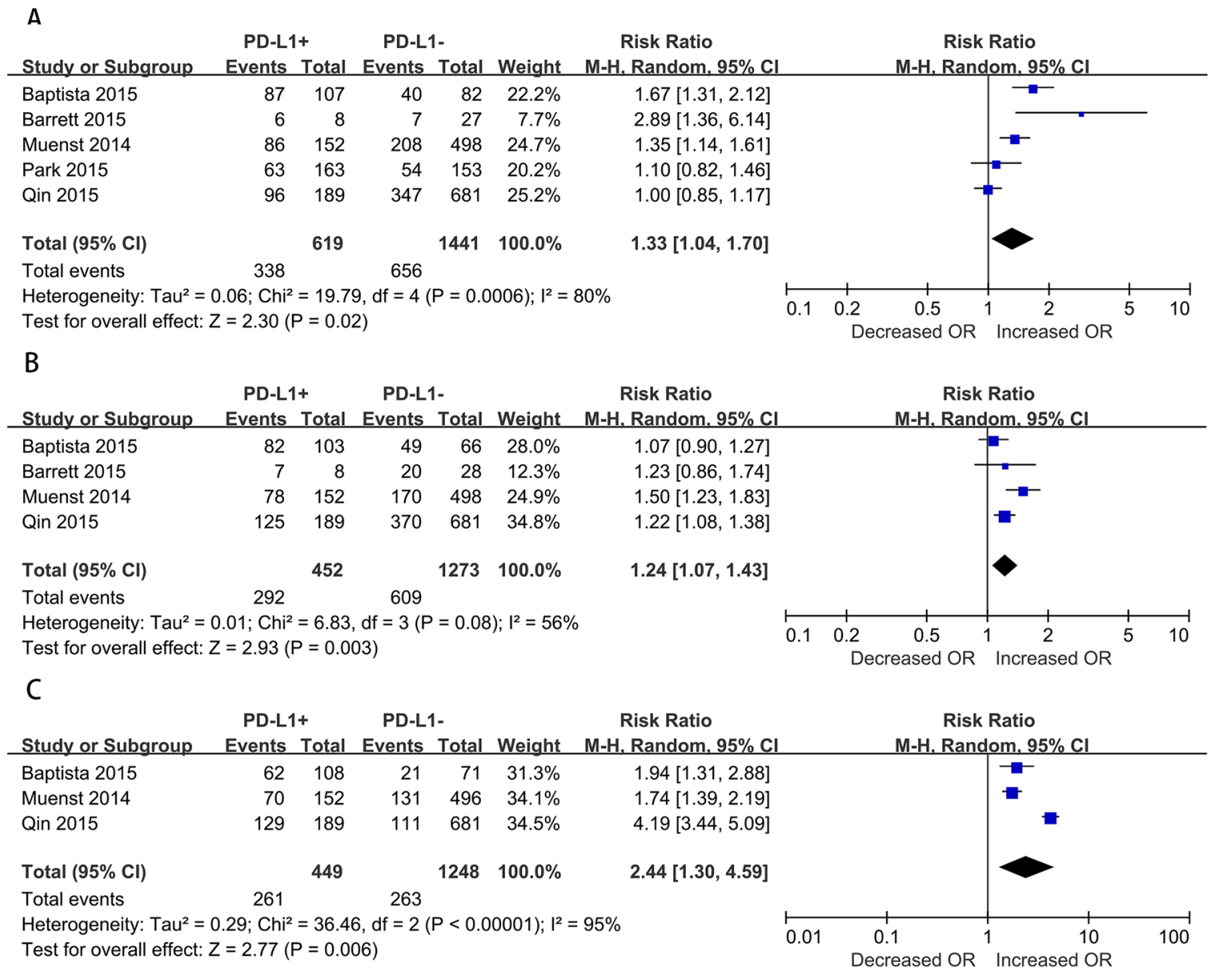

Fig 3. Forest plots of studies evaluating the association between PD-L1 and clinical parameters in breast cancer. (A) Lymph node metastasis (positive versus negative). (B) Nuclear grade (3, 4 versus 1, 2). (C) ER status (negative versus positive).

doi:10.1371/journal.pone.0156323.g003 


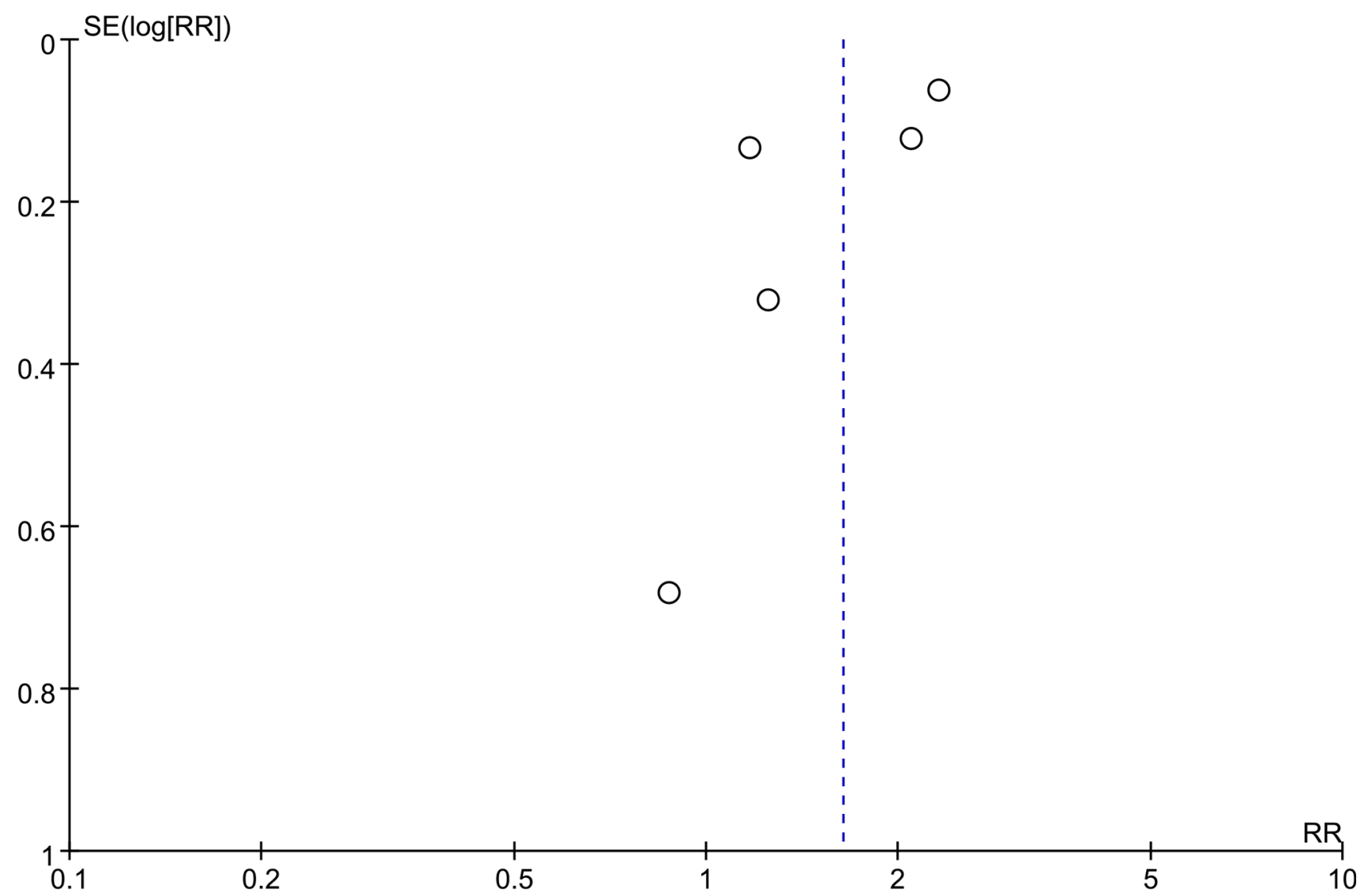

Fig 4. Funnel plots for all of the included studies reported in this meta-analysis.

doi:10.1371/journal.pone.0156323.g004

2.45 (95\% CI, 1.31-4.60; Fig 3C). However, no significant relationship was observed between PD-L1 overexpression and other clinical characteristics such as tumor size; TNM stages; and expression of PR, Ki67, and Her2 in breast cancer due to insufficient data. Furthermore, high PD-L1 expression was significantly associated with lymph node metastasis, poor nuclear grade, and negative ER expression.

\section{Publication bias}

The funnel plot did not indicate any evidence of publication bias (Fig 4).

\section{Discussion}

At present, the relationship between PD-L1 and clinical outcomes in breast cancer patients remains unclear. In this study, we focused on the prognostic utility of PD-L1 in breast cancer and its relationship with the clinicopathological features of breast cancer patients. To our knowledge, this is the first meta-analysis to discuss this topic. From our analysis, we observed that the pooled RR value for mortality in breast cancer patients was 1.64 (95\% CI, 1.14-2.34), which suggests that patients with positive/higher PD-L1 expression have significantly poorer outcomes, as compared to patients with negative/lower PD-L1 expression. To avoid bias caused by the follow-up duration after surgery, we assessed the $\mathrm{MR}_{10 y e a r s}$ and found that the pooled RR of mortality 10 years after surgery was 2.53 (95\% CI, 1.78-3.59), which is consistent with the abovementioned results. 
Several antibodies that inhibit the PD-1 pathway (by blocking either PD-1 or PD-L1) are being developed for clinical use in various tumor types and clinical settings [22]. Researchers have observed that treatment with anti-PD-L1 antibody ibrutinib (a covalent inhibitor of Bruton's tyrosine kinase) suppresses tumor growth in mouse models of triple-negative breast cancer (TNBC) [23]. Moreover, several clinical trials are ongoing wherein the PD-1/PD-L1

pathway is targeted with MEDI4736 or MPDL3280A, which are monoclonal antibodies against PD-L1. Notably, two early phase I trials involving PD-1/PD-L1 blockade (with pembrolizumab or MPDL3280A) in TNBC have demonstrated overall response rates of $15-20 \%[24,25]$.

According to the preliminary report of a phase 1A trial (MPDL3280A) presented at the annual meeting of the American Association for Cancer Research's in 2015, objective responses were noted in $24 \%$ of patients (95\% CI 8-47); $10 \%$ patients showed complete responses and $14 \%$ patients showed partial responses; $29 \%$ patients had progression-free survival of 24 weeks or longer, after following up for 40 weeks[26]. Thus, treatment with PD-L1 could be an option in TNBC, cause all theses trails have been conducted in patients with TNBC, but not in other breast cancer subtypes. These findings suggest that the high expression of PD-L1 indicates a poor outcome and that treatment with anti-PD-L1 antibodies should be attempted in patients with breast carcinoma in future clinical trials.

While examining the relationship between PD-L1 expression and clinicopathological features, we observed that the pooled RR was significantly associated with lymph node metastasis, poor nuclear grade, and negative ER expression. These findings are consistent with the results of other studies, including the study by Mittendorf et al, wherein PD-L1+ carcinoma cells were observed more often in TNBC than in other breast cancer subtypes [6] and the study of Wimberly et al., wherein PD-L1 expression correlated with the lack of ER expression [27]. No previous studies have definitely indicated that nuclear grade and lymph node metastasis are related to PD-L1 expression in breast cancer, although some articles on other cancers have described these relationships for other cancer[18, 28, 29]. A large number of metastatic lymph nodes, poorer nuclear grade, and negative ER expression all indicate a poor outcome. These findings support the sensitivity and specificity of PD-L1 in the predicting clinical survival in patients with breast carcinoma. Thus, part of the patients with metastatic lymph nodes, poorer nuclear grade, and negative ER expression may benefit from anti-PD-L1 therapy, which would improve their prognosis and help improve the OS rate. However, wheter PD-L1 expression is a predictive marker for the response to anti-PD-L1 therapy in breast cancer patients is still a question need to be solved.

Three study were excluded from this meta-analysis because PD-L1 expression were detected using DNA microarray[30,31] or fluorescent RNAscope paired-primer assay[32]. Clearly, comparing IHC-based protein expression with microarray-based gene-expression levels can lead to quite distinct conclusions. For example, DNA microarray-based measurements quantify expression levels in tumor cells, non-tumor cells, and infiltrating immune cells, while our present study focused on the expression of PD-L1 in tumor cells. Sabatier et al and Schalper et al concluded that PD-L1 upregulation was associated with better survival in patients with breast cancer especially basal-like breast cancer (express genes characteristic of the outer or basally located epithelial layer of the mammary gland) [31-33], while Bertucci et al considered PD-L1 overexpression in inflammatory breast cancer orrelated with better response to chemotherapy[30]. The differences in results between these studies may be mainly due to differences in the detection method used and the subtype of breast cancer.

Nevertheless, the present study has certain limitations. First, PD-L1 is a novel target that has not been extensively studied; in fact, most of the included studies had a relatively small sample size and could not be compared. Second, the antibodies used in each study were different, as were the sensitivities of the antibodies, the experimental procedures and 
immunohistochemical reagents, the scoring method, the cut-off value for PD-L1 overexpression, and the study end points varied. Collectively, these factors could lead to a high degree of heterogeneity. Third, potential factors such as the therapeutic strategy, patient age, and BMI, which were not considered in the meta-analysis may have impacted on our results, even if similar inclusion criteria were used for each study.

In conclusion, our findings revealed that PD-L1 should be considered as a prognostic indicator of poor survival in patients with breast cancer. Since high PD-L1 expression is associated to canonical prognostic factors of breast cancer, patients with positive PD-L1 expression may present with more extensive lymph node metastasis and poor nuclear grade. Even though PD-L1 expression does not add so much new insight for the prognostic characterization of the patient but it can be used for the selection of patients candidate to immunotherapy. Hence, in addition to endocrine therapy or chemotherapy, such patients may benefit from anti PD-L1 therapy. Furthermore, additional studies are needed from multiple centers with large sample sizes and detailed follow-up, in order to study the role of the PD-1/PD-L1 pathway in breast cancer.

\section{Supporting Information}

\section{S1 File. Completed 2009 PRISMA Checklist. (DOC)}

S2 File. A full list of excluded articles and their reasons for exclusion. (DOC)

\section{Author Contributions}

Conceived and designed the experiments: CL TH. Performed the experiments: YG PY. Analyzed the data: YG PY ZL. Contributed reagents/materials/analysis tools: YM SW XY. Wrote the paper: YG PY.

\section{References}

1. Torre LA, Bray F, Siegel RL, Ferlay J, Lortet-Tieulent J, Jemal A. Global cancer statistics, 2012. CA: a cancer journal for clinicians. 2015; 65(2):87-108. doi: 10.3322/caac.21262 PMID: 25651787.

2. Goldhirsch A, Winer EP, Coates AS, Gelber RD, Piccart-Gebhart M, Thurlimann B, et al. Personalizing the treatment of women with early breast cancer: highlights of the St Gallen International Expert Consensus on the Primary Therapy of Early Breast Cancer 2013. Annals of oncology: official journal of the European Society for Medical Oncology / ESMO. 2013; 24(9):2206-23. doi: 10.1093/annonc/mdt303 PMID: 23917950; PubMed Central PMCID: PMC3755334.

3. Coates AS, Winer EP, Goldhirsch A, Gelber RD, Gnant M, Piccart-Gebhart M, et al. Tailoring therapies -improving the management of early breast cancer: St Gallen International Expert Consensus on the Primary Therapy of Early Breast Cancer 2015. Annals of Oncology. 2015; 26(8):1533-46. doi: 10.1093/ annonc/mdv221 PMID: 25939896

4. Mohammed ZM, Going JJ, Edwards J, Elsberger B, Doughty JC, McMillan DC. The relationship between components of tumour inflammatory cell infiltrate and clinicopathological factors and survival in patients with primary operable invasive ductal breast cancer. British journal of cancer. 2012; 107 (5):864-73. doi: 10.1038/bjc.2012.347 PMID: 22878371; PubMed Central PMCID: PMCPMC3426752.

5. Zou W, Chen L. Inhibitory B7-family molecules in the tumour microenvironment. Nat Rev Immunol. 2008; 8(6):467-77. doi: 10.1038/nri2326 PMID: 18500231.

6. Mittendorf EA, Philips AV, Meric-Bernstam F, Qiao N, Wu Y, Harrington S, et al. PD-L1 expression in triple-negative breast cancer. Cancer immunology research. 2014; 2(4):361-70. Epub 2014/04/26. doi: 10.1158/2326-6066.cir-13-0127 PMID: 24764583; PubMed Central PMCID: PMCPMC4000553.

7. Bertucci F, Finetti P, Mamessier E, Pantaleo MA, Astolfi A, Ostrowski J, et al. PDL1 expression is an independent prognostic factor in localized GIST. Oncoimmunology. 2015; 4(5):e1002729. doi: 10. 1080/2162402X.2014.1002729 PMID: 26155391; PubMed Central PMCID: PMCPMC4485716. 
8. Gatalica Z, Snyder C, Maney T, Ghazalpour A, Holterman DA, Xiao N, et al. Programmed cell death 1 (PD-1) and its ligand (PD-L1) in common cancers and their correlation with molecular cancer type. Cancer epidemiology, biomarkers \& prevention: a publication of the American Association for Cancer Research, cosponsored by the American Society of Preventive Oncology. 2014; 23(12):2965-70. Epub 2014/11/14. doi: 10.1158/1055-9965.epi-14-0654 PMID: 25392179.

9. Tarhini AA, Zahoor H, Yearley JH, Gibson C, Rahman Z, Dubner R, et al. Tumor associated PD-L1 expression pattern in microscopically tumor positive sentinel lymph nodes in patients with melanoma. Journal of translational medicine. 2015; 13:319. doi: 10.1186/s12967-015-0678-7 PMID: 26419843; PubMed Central PMCID: PMCPMC4589168.

10. Thoma C. Prostate cancer: PD-L1 expression is common and indicates poor prognosis. Nat Rev Urol. 2016; 13(1):5. doi: 10.1038/nrurol.2015.287 PMID: 26620611.

11. Zhu L, Jing S, Wang B, Wu K, Shenglin MA, Zhang S. Anti-PD-1/PD-L1 Therapy as a Promising Option for Non-Small Cell Lung Cancer: a Single arm Meta-Analysis. Pathology oncology research: POR. 2015. doi: 10.1007/s12253-015-0011-z PMID: 26552662.

12. Bigelow E, Bever KM, Xu H, Yager A, Wu A, Taube J, et al. Immunohistochemical staining of B7-H1 (PD-L1) on paraffin-embedded slides of pancreatic adenocarcinoma tissue. Journal of visualized experiments: JoVE. 2013;(71: ). doi: 10.3791/4059 PMID: 23328703; PubMed Central PMCID: PMCPMC3582653.

13. Muenst S, Schaerli AR, Gao F, Daster S, Trella E, Droeser RA, et al. Expression of programmed death ligand 1 (PD-L1) is associated with poor prognosis in human breast cancer. Breast cancer research and treatment. 2014; 146(1):15-24. Epub 2014/05/21. doi: 10.1007/s10549-014-2988-5 PMID: 24842267; PubMed Central PMCID: PMCPmc4180714.

14. Baptista MZ, Sarian LO, Derchain SF, Pinto GA, Vassallo J. Prognostic significance of PD-L1 and PDL2 in breast cancer. Human pathology. 2015. Epub 2015/11/07. doi: 10.1016/j.humpath.2015.09.006 PMID: 26541326.

15. Barrett MT, Anderson KS, Lenkiewicz E, Andreozzi M, Cunliffe HE, Klassen CL, et al. Genomic amplification of 9p24.1 targeting JAK2, PD-L1, and PD-L2 is enriched in high-risk triple negative breast cancer. Oncotarget. 2015; 6(28):26483-93. Epub 2015/09/01. doi: 10.18632/oncotarget.4494 PMID: 26317899.

16. Park IH, Kong SY, Ro JY, Kwon Y, Kang JH, Mo HJ, et al. Prognostic Implications of Tumor-Infiltrating Lymphocytes in Association With Programmed Death Ligand 1 Expression in Early-Stage Breast Cancer. Clinical breast cancer. 2015. Epub 2015/09/14. doi: 10.1016/j.clbc.2015.07.006 PMID: 26364145.

17. Qin T, Zeng YD, Qin G, Xu F, Lu JB, Fang WF, et al. High PD-L1 expression was associated with poor prognosis in 870 Chinese patients with breast cancer. Oncotarget. 2015; 6(32):33972-81. Epub 2015/ 09/18. doi: 10.18632/oncotarget.5583 PMID: 26378017.

18. Xu F, Xu L, Wang Q, An G, Feng G, Liu F. Clinicopathological and prognostic value of programmed death ligand-1 (PD-L1) in renal cell carcinoma: a meta-analysis. International journal of clinical and experimental medicine. 2015; 8(9):14595-603. PMID: 26628942; PubMed Central PMCID: PMCPMC4658831.

19. Stang A. Critical evaluation of the Newcastle-Ottawa scale for the assessment of the quality of nonrandomized studies in meta-analyses. Eur J Epidemiol. 2010; 25(9):603-5. doi: 10.1007/s10654-0109491-z PMID: 20652370.

20. Tierney JF, Stewart LA, Ghersi D, Burdett S, Sydes MR. Practical methods for incorporating summary time-to-event data into meta-analysis. Trials. 2007; 8:16. doi: 10.1186/1745-6215-8-16 PMID: $17555582 ;$ PubMed Central PMCID: PMCPMC1920534.

21. Egger M, Davey Smith G, Schneider M, Minder C. Bias in meta-analysis detected by a simple, graphical test. BMJ. 1997; 315(7109):629-34. PMID: 9310563; PubMed Central PMCID: PMCPMC2127453.

22. Homet Moreno B, Ribas A. Anti-programmed cell death protein-1/ligand-1 therapy in different cancers. British journal of cancer. 2015; 112(9):1421-7. doi: 10.1038/bjc.2015.124 PMID: 25856776; PubMed Central PMCID: PMCPMC4453674.

23. Sagiv-Barfi I, Kohrt HE, Czerwinski DK, Ng PP, Chang BY, Levy R. Therapeutic antitumor immunity by checkpoint blockade is enhanced by ibrutinib, an inhibitor of both BTK and ITK. Proceedings of the National Academy of Sciences of the United States of America. 2015; 112(9):E966-72. Epub 2015/03/ 03. doi: 10.1073/pnas.1500712112 PMID: 25730880; PubMed Central PMCID: PMCPmc4352777.

24. Nanda R CL, Dees EC, Berger R, Gupta S, Geva R, Pusztai L, Dolled-Filhart M, Emancipator K, Gonzalez E, Houp J, Pathiraja K, Karantza V, lannone R, Gause C, Cheng J, et al. A phase lb study of pembrolizumab (MK-3475) in patients with advanced triple-negative breast cancer. San Antonio Breast Cancer Symposium. 2014;(San Antonio TX). 
25. Emens LA BF, Cassier P, DeLord J-P, Eder JP, Shen X, Xiao Y, Wang Y, Hedge PS, Chen DS, Krop I. Inhibition of PD-L1 by MPDL3280A leads to clinical activity in patients with metastatic triple-negative breast cancer. San Antonio Breast Cancer Symposium. 2014; (San Antonio TX).

26. Gibson J. Anti-PD-L1 for metastatic triple-negative breast cancer. The Lancet Oncology. 2015; 16(6): e264. Epub 2015/05/06. doi: 10.1016/s1470-2045(15)70208-1 PMID: 25936988.

27. Wimberly H, Brown JR, Schalper K, Haack H, Silver MR, Nixon C, et al. PD-L1 Expression Correlates with Tumor-Infiltrating Lymphocytes and Response to Neoadjuvant Chemotherapy in Breast Cancer. Cancer immunology research. 2014. Epub 2014/12/21. doi: 10.1158/2326-6066.cir-14-0133 PMID: 25527356.

28. Heeren AM, de Boer E, Bleeker MC, Musters RJ, Buist MR, Kenter GG, et al. Nodal metastasis in cervical cancer occurs in clearly delineated fields of immune suppression in the pelvic lymph catchment area. Oncotarget. 2015; 6(32):32484-93. doi: 10.18632/oncotarget.5398 PMID: 26431490.

29. Oliveira-Costa JP, de Carvalho AF, da Silveira da GG, Amaya P, Wu Y, Park KJ, et al. Gene expression patterns through oral squamous cell carcinoma development: PD-L1 expression in primary tumor and circulating tumor cells. Oncotarget. 2015; 6(25):20902-20. Epub 2015/06/05. doi: 10.18632/ oncotarget.3939 PMID: 26041877; PubMed Central PMCID: PMCPmc4673238.

30. Bertucci F, Finetti P, Colpaert C, Mamessier E, Parizel M, Dirix L, et al. PDL1 expression in inflammatory breast cancer is frequent and predicts for the pathological response to chemotherapy. Oncotarget. 2015; 6(15):13506-19. PMID: WOS:000359009400048.

31. Sabatier R, Finetti P, Mamessier E, Adelaide J, Chaffanet M, Ali HR, et al. Prognostic and predictive value of PDL1 expression in breast cancer. Oncotarget. 2015; 6(7):5449-64. Epub 2015/02/12. doi: 10. 18632/oncotarget.3216 PMID: 25669979; PubMed Central PMCID: PMCPmc4467160.

32. Schalper KA, Velcheti V, Carvajal D, Wimberly H, Brown J, Pusztai L, et al. In Situ Tumor PD-L1 mRNA Expression Is Associated with Increased TILs and Better Outcome in Breast Carcinomas. Clinical Cancer Research. 2014; 20(10):2773-82. PMID: WOS:000336720200028. doi: 10.1158/1078-0432.CCR13-2702

33. Perou Charles M., Sùrlie Therese, Eisen Michael B., van de Rijn Matt, Jeffreyk Stefanie S., Rees Christian A., et al. Molecular portraits of human breast tumours. Nature. 2000; 406(6797):747-52. doi: 10. 1038/35021093 PMID: 10963602 